PHYSICAL REVIEW B 95, 159904(E) (2017)

\title{
Publisher's Note: Evidence of density waves in single-crystalline nanowires of pyrochlore iridates
} [Phys. Rev. B 95, 125436 (2017)]

\section{Abhishek Juyal, Amit Agarwal, and Soumik Mukhopadhyay}

(Received 30 March 2017; published 7 April 2017)

DOI: 10.1103/PhysRevB.95.159904

This paper was published online on 28 March 2017 with an omission of two curves in panel (d) in Fig. 4. The panel has been replaced as of 31 March 2017. The panel is correct in the printed version of the journal. 